\title{
Digital Image Analysis of CD8+ and CD3+ Tumor-Infiltrating Lymphocytes in Tongue Squamous Cell Carcinoma
}

This article was published in the following Dove Press journal: Cancer Management and Research

\author{
Yenlin Huang $\mathbb{D}^{1,2}$ \\ Chieh Lin ${ }^{3}$ \\ Huang-Kai Kao ${ }^{2,4}$ \\ Shao-Yu Hung 4 \\ Hung-Ju Ko ${ }^{2}$ \\ Yu-Chen Huang ${ }^{5}$ \\ Yu-Liang Chang ${ }^{5}$ \\ Kai-Ping Chang (iD) 2,6 \\ 'Department of Anatomic Pathology, \\ Chang Gung Memorial Hospital, Taoyuan, \\ Taiwan; ${ }^{2}$ College of Medicine, Chang \\ Gung University, Taoyuan, Taiwan; \\ ${ }^{3}$ Department of Nuclear Medicine, Chang \\ Gung Memorial Hospital, Taoyuan, \\ Taiwan; ${ }^{4}$ Department of Plastic and \\ Reconstructive Surgery, Chang Gung \\ Memorial Hospital, Taoyuan, Taiwan; \\ ${ }^{5}$ Department of Oral \& Maxillofacial \\ Surgery, Chang Gung Memorial Hospital, \\ Taoyuan, Taiwan; ' Department of \\ Otolaryngology - Head \& Neck Surgery, \\ Chang Gung Memorial Hospital, Taoyuan, \\ Taiwan
}

Purpose: The presence of CD8+ tumor-infiltrating lymphocytes (TILs) has been reported to be associated with treatment outcomes in many types of solid tumors. However, the results vary due to the various methods of visual estimation and subjective interpretation. The current study is the first to use digital image analyses to evaluate the density of CD8 +/CD3+ TILs in tongue squamous cell carcinoma (TSCC).

Patients and Methods: From 2005 to 2015, a cohort of 258 TSCC patients were consecutively enrolled in this study. After immunohistochemistry on representative sections, the density of $\mathrm{CD} 8+/ \mathrm{CD} 3+$ TILs at tumor invasive margins was evaluated by digital image analysis. The subjects were stratified by the median values of CD3+ cell density, CD8+ cell density, $\mathrm{CD} 8 / \mathrm{CD} 3$, and scores $(0,1$, and 2$)$ to demonstrate the association with various clinicopathological manifestations.

Results: Low CD8+ TIL counts were associated with advanced tumor stages $(p=0.034)$, and low $\mathrm{CD} 8 / \mathrm{CD} 3$ ratios were associated with perineural invasion $(p=0.043)$. Both parameters were also associated with increased tumor depths ( $p=0.034$ and 0.004 , respectively). Univariate analyses revealed that advanced tumor stages, perineural invasion, extranodal extension, tumor depth, lower CD8 counts, and lower scores (score 0 vs 2) were associated with poorer overall survival, and multivariate analysis further indicated that extranodal extension and low scores (score 0 vs 2 ) were both independent factors for overall survival ( $\mathrm{p}<0.0001$ and $\mathrm{p}=0.0369$, respectively).

Conclusion: The use of digital image analysis to assess CD8+ TILs at the invasive margins provides an objective indicator of prognoses including overall survival.

Keywords: oral cavity, squamous cell carcinoma, oral cancer, SCC, tumor-infiltrating lymphocyte, TIL

\section{Introduction}

Oral cavity squamous cell carcinoma (OSCC) is a key cause of mortality in patients with head and neck cancer worldwide and the fifth most common type of cancer among males in Taiwan. ${ }^{1}$ Furthermore, tongue squamous cell carcinoma (TSCC) is the most common malignancy in the oral cavity worldwide. ${ }^{1-3}$ The 5 -year overall survival (OS) for TSCC remains $50 \sim 60 \%$ in spite of aggressive multimodal treatments including radical surgery, concurrent chemoradiotherapy and/or adjuvant chemotherapy. ${ }^{4}$ Several previous studies have attempted to predict the OS of OSCC patients by evaluating the density of tumor-infiltrating lymphocytes (TILs) in OSCC tumors in various parts of the oral cavity: ${ }^{5-8}$ However, the heterogeneous anatomic
Correspondence: Kai-Ping Chang Department of Otolaryngology - Head \& Neck Surgery, Chang Gung Memorial Hospital, No. 5, Fu-Hsing Street, KweiShan, Taoyuan 333005, Taiwan

Tel $+886-3-328 I 200$ ext 3972

Fax +886-3-397936।

Email dr.kpchang@gmail.com 
location of OSCC and the lack of consistent evaluation methods has delayed the generation of a solid conclusion.

Among the different subsets of leukocytes that play distinct roles in the tumor microenvironment, CD8+ TILs have been shown to play a major role in antitumor immunity by releasing cytotoxic granules containing perforin, granulysin and granzymes to eliminate the encountered tumor cells, and a higher number of CD8+ TILs have been noted to be associated with better prognosis in several types of cancer, including breast, colorectal, and head and neck cancer. $^{6-11}$ Lee et al used cytokeratin immunohistochemistry to separate the epithelial from the stromal compartment in tissue microarrays from 91 patients (corresponding to 182 samples). ${ }^{12}$ The authors found that high infiltrating CD8+ TILs (that is, in the epithelial compartment) were associated with an inferior survival status in patients treated with adjuvant radiotherapy. The authors hypothesized that radiotherapy might alter the tumor microenvironment. Additionally, previous studies had several limitations. First, the association of CD8+ TILs with clinicopathological features and their clinical impacts on OS might vary due to the heterogeneity of the tumor location in the oral cavity. Second, manual methods of evaluating the density of CD8+ TILs in the tumor microenvironment might lead to inconsistent intra- and interobserver reproducibility. Although one study investigated the role of CD8+ TILs in TSCC tumors, ${ }^{5}$ it was limited by a small number of patients and a manual method to enumerate CD8+ TILs. To the best of our knowledge, no application of digital image analysis for studying the role of tumor-infiltrating lymphocytes in a large cohort of TSCC cases has been reported.

The aim of this study was to use digital image analyses to quantify the density of CD3+ and CD8+ TILs by examining the invasive margins in a large cohort of TSCC patients. The density of CD3+ and CD8+ TILs in histopathological examination was stratified by the median values of $\mathrm{CD} 3+$ and CD8+ TILs based on the density $\left(\mathrm{TILs} / \mathrm{mm}^{2}\right)$. The association of the $\mathrm{CD} 8$ to $\mathrm{CD} 3$ ratio with clinicopathological factors and survival was further analyzed to demonstrate the potential roles of $\mathrm{CD} 8+$ and CD3+ TILs in TSCC tumors and patients.

\section{Patients and Methods}

\section{Patient Characteristics and Clinical}

\section{Specimens}

In this study, we retrospectively enrolled consecutive patients with TSCC who were diagnosed at Chang Gung
Memorial Hospital between September 2005 and December 2015. The patients were defined as betel nut chewers if they chewed 2 or more betel nuts daily for at least 1 year, as cigarette smokers if they smoked every day for at least 1 year, and as alcohol drinkers if they consumed an alcoholic beverage 1 or more times per week for at least 6 months. Patients with at least one of the following conditions were considered ineligible for study inclusion: other concomitant primary cancer (synchronous or metachronous), recurrent disease, any prior history of malignancy, or treatment with neoadjuvant therapy. This study was approved by the institutional review board. The patients underwent standard preoperative work-ups per institutional guidelines, including a detailed medical history, complete physical examination, blood tests, computed tomography or magnetic resonance imaging scans of the head and neck, chest radiographs, bone scan, and abdominal ultrasound. The primary tumors were excised with adequate margins using intraoperative frozen section controls transorally or via lip splitting with a mandibular swing approach, and neck dissections were simultaneously performed in level I-III (for clinical N0 neck disease) or level I-V (for clinical $\mathrm{N}+$ disease). Surgical defects were primarily closed or immediately reconstructed, if necessary, by plastic surgeons using local, pedicled, or free flaps. Following surgical treatment, the pathological TNM classification of all tumors was established according to the American Joint Committee on Cancer Staging Manual (2010). Patients with stage pT4 tumors, pathologically positive lymph nodes, or microscopic close margins $(<5 \mathrm{~mm})$ were administered adjuvant radiotherapy, and concurrent chemoradiation therapy was administered to patients with extranodal extension, positive surgical margins, or multiple positive lymph node metastases. The chemotherapy was a cisplatin-based regimen, and the total radiation dose for radiotherapy was usually $\sim 70$ Gy. The prescribed dose was delivered 2 Gy per day and 5 days per week. After discharge, all patients underwent regular follow-up visits every 2 months for the first year, every 3 months for the second year, and every 6 months thereafter. ${ }^{4}$

\section{Immunohistochemistry and Digital Image Analysis}

Hematoxylin and eosin (HE)-stained slides were obtained from the archives of the Department of Anatomic Pathology, 
Chang Gung Memorial Hospital (Taoyuan, Taiwan). The invasive margin was defined as a region centered on the border separating the host tissue from the tumor cells, with an extent of $1 \mathrm{~mm} .{ }^{13}$ An automatic slide scanner was unfortunately not available at the time of conducting this study; therefore, four representative fields (region of interest, ROI, corresponding to $0.58 \mathrm{~mm}^{2}$ ) in each section were selected at $200 \mathrm{x}$ magnification per field after an initial screen under a microscope at low power (40x). The representative images photographed at low magnification (20x) are shown in Figure 1. The corresponding sections were cut at $3 \mu \mathrm{m}$ thickness and incubated with mouse anti-human monoclonal CD3 (1:100; Novocastra LN10, Leica Microsystems, Wetzlar, Germany) and CD8 (1:100; Novocastra 4B11, Leica Microsystems, Wetzlar, Germany) antibodies using a Leica BOND-MAX autostainer (Leica Microsystems, Wetzlar, Germany). Isotype-matched mouse monoclonal antibodies were used as negative controls.

Images were captured in four different areas with the highest densities of CD3+ and CD8+ cells along the invasive margin under $200 \mathrm{X}$ magnification, equivalent to 0.58 $\mathrm{mm}^{2}$ with a 22-mm field of ocular view. The ROI images were captured under the Olympus BX50 microscope (Olympus, Tokyo, Japan). The numbers of CD3 + and CD8 + TILs were identified using ImagePro software and then averaged for each slide. The results are shown as the mean number per $\mathrm{mm}^{2}$ (Figure $1 \mathrm{E}-\mathrm{H}$ ). The densities of CD3+ and CD8+ cells (number of positive cells per $\mathrm{mm} 2$ ) were analyzed using Image-Pro Premier 9.1 (Media Cybernetics, Rockville, MD, USA).

\section{Statistical Analysis}

Chi-square and Wilcoxon tests were used to compare the clinicopathological characteristics in TSCC patients. All patients underwent follow-up evaluations at our outpatient clinic until June 2018 or death. Survival analyses were plotted using the Kaplan-Meier method, and differences were evaluated using the Log rank test. Cox proportional hazards models were employed to define the independent factors for OS. Statistical analyses were performed using SAS software (version 9.3; SAS Institute, Cary, NC). The statistical significance of all $p$-values was set at $p<0.05$.

\section{Results}

\section{Patient Characteristics and Clinicopathological Data}

During the study period, 258 patients consisting of 222 $(86.0 \%)$ males and $36(14.0 \%)$ females were enrolled. Of the enrolled patients, $73.3 \%$ were smokers, $62.8 \%$ were alcohol consumers, and $73.6 \%$ were betel nut chewers. The patient age at diagnosis ranged from 21.9 to 83.9 years old (mean, $52.7 \pm 11.8$ ); CD3 + cell density ranged from 309.4 to $4594.8 / \mathrm{mm}^{2}$ (mean, $2059.0 \pm 868.7$ ); CD8+ cell density ranged from 151.7 to $3491.3 / \mathrm{mm}^{2}$ (mean, $1253.8 \pm 693.3)$; and the CD8 to CD3 ratio ranged from 0.16 to 1.02 (mean, $0.59 \pm 0.16$ ). The most common TNM stage was IV (34.5\%), followed by II (24.8\%), I (24\%) and III $(16.7 \%)$. All patient characteristics are described in Table 1. Dichotomization of the patients by CD3+ cell density, CD8+ cell density and the CD8 to CD3 ratio was performed using the median values as cut-offs, which were $2016 / \mathrm{mm}^{2}$ for CD3+ cell density, $1125 / \mathrm{mm}^{2}$ for $\mathrm{CD} 8+$ cell density and 0.592 for $\mathrm{CD} 8 / \mathrm{CD} 3$.

\section{Association of Clinicopathological Manifestations with CD3+ TILs, CD8+ TILs, CD8/CD3, and Scores}

The subjects were stratified by the median values of $\mathrm{CD} 3+$ cell density, CD8+ cell density, CD8/CD3, and scores $(0,1$, and 2) to demonstrate the association with various clinicopathological manifestations. Cases with $\mathrm{CD} 8+$ cell counts and $\mathrm{CD} 8 / \mathrm{CD} 3$ ratio lower than the median values were given a score of 0 , and cases with CD8+ cell counts and $\mathrm{CD} 8 / \mathrm{CD} 3$ ratio higher than the median values were given a score of 2. The related details are shown in Table 2 . Decreased CD8+ cell density and CD8/CD3 values were associated with a higher $\mathrm{pT}$ classification $(p=0.034$ and 0.043 , respectively) and positive perineural invasion ( $p=$ 0.449 and 0.043 , respectively). More interestingly, lower CD8+ cell densities, CD8/CD3 values and scores were associated with increased tumor depths $(p=0.034,0.004$, and 0.018 , respectively). These results indicate that lower CD8+ cell densities, CD8/CD3 values and scores may be related to more aggressive TSCC tumors in this retrospective cohort.

\section{Association of CD3+ TILs, CD8+ TILs, CD8/CD3, and Scores with OS}

The mean follow-up period was $4.5 \pm 3.2$ years, and the survival analyses for the entire cohort of 258 patients demonstrated that the five-year OS was $70.6 \%$. The OS probability analysis revealed the five-year OS incidence for patients in subgroups stratified by overall stages, perineural invasion, extranodal extension, tumor depth $(<5 \mathrm{~mm}$ or $\geq 5 \mathrm{~mm}), \mathrm{CD} 3+$ cell densities, $\mathrm{CD} 8+$ cell densities, and the $\mathrm{CD} 8 / \mathrm{CD} 3$ ratio 

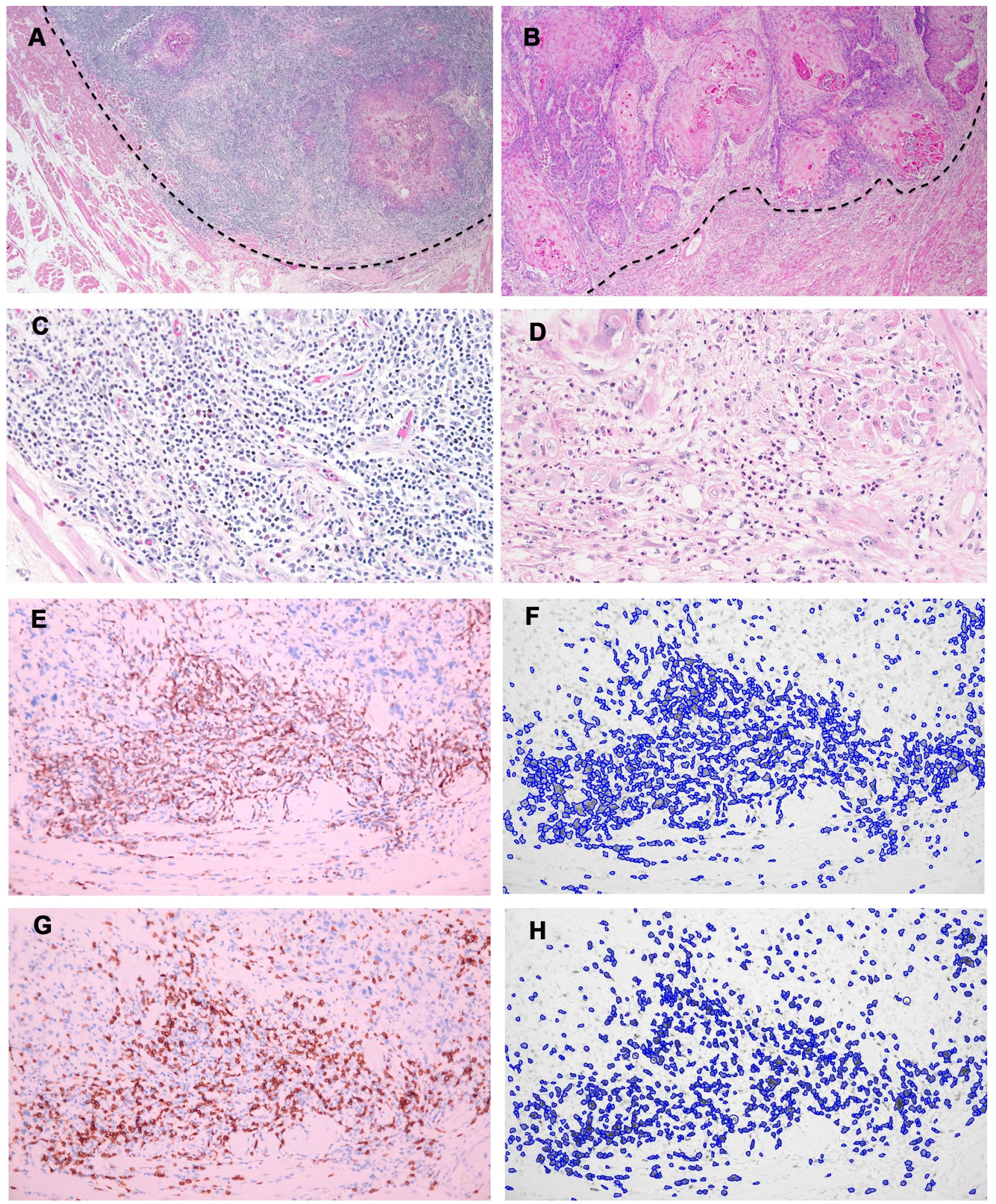

Figure I (A-D) The invasive margin of two representative cases of tongue squamous cell carcinoma. (hematoxylin and eosin stain; 20x (A, B) and 200x (C, D) original magnification). (E-H) A case example of the tumor-infiltrating lymphocyte (TIL) counting strategy. Examples of images from the same region of interest under 200x magnification from used to extract total positive staining counts in Image Pro. The total TIL counts were calculated based on the transfer of data from CD3 and CD8 IHC images to black-and-white grid format. (E, CD3 IHC image; F, CD3 Image-Pro image; G, CD8 IHC image, H, CD8 Image-Pro image; 200x original magnification). 
Table I Clinical, Pathological, and Laboratory Characteristics of Patients

\begin{tabular}{|c|c|c|}
\hline Characteristics & Number & Percentage, \% \\
\hline \multicolumn{3}{|l|}{ Sex } \\
\hline Male & 222 & 86.0 \\
\hline Female & 36 & 14.0 \\
\hline \multicolumn{3}{|l|}{ PT classification } \\
\hline TI & 70 & 27.1 \\
\hline $\mathrm{T} 2$ & 102 & 39.5 \\
\hline T3 & 29 & 11.3 \\
\hline T4 & 57 & 22.1 \\
\hline \multicolumn{3}{|l|}{ pN classification } \\
\hline No & 165 & 64.0 \\
\hline $\mathrm{NI}$ & 33 & 12.8 \\
\hline N2 & 60 & 23.2 \\
\hline \multicolumn{3}{|l|}{$\begin{array}{l}\text { Overall pathological } \\
\text { stage }\end{array}$} \\
\hline 1 & 62 & 24.0 \\
\hline II & 64 & 24.8 \\
\hline III & 43 & 16.7 \\
\hline IV & 89 & 34.5 \\
\hline \multicolumn{3}{|l|}{ Alcohol consumption } \\
\hline No & 96 & 37.2 \\
\hline Yes & 162 & 62.8 \\
\hline \multicolumn{3}{|l|}{ Betel nut chewing } \\
\hline No & 68 & 26.4 \\
\hline Yes & 190 & 73.6 \\
\hline \multicolumn{3}{|l|}{ Smoking } \\
\hline No & 69 & 26.7 \\
\hline Yes & 189 & 73.3 \\
\hline Characteristics & Number & Mean \pm SD (Max, Min) \\
\hline Age (years) & 258 & $52.7 \pm$ II.8 $(83.9,21.9)$ \\
\hline CD3 $\left(/ \mathrm{mm}^{2}\right)$ & 258 & $2059.0 \pm 868.7(4594.8,309.4)$ \\
\hline CD8 $\left(/ \mathrm{mm}^{2}\right)$ & 258 & $1253.8 \pm 693.3(349 \mid .3,151.7)$ \\
\hline CD8/CD3 & 258 & $0.59 \pm 0.16(1.02,0.16)$ \\
\hline
\end{tabular}

(Figure 2). In addition to the significant difference by overall pathological stages, perineural invasion, extranodal extension, and tumor depths, the current study further demonstrated that the OS rates in for patients in subgroups stratified by CD3+ cell densities $>2016 / \mathrm{mm}^{2}$ and $\leq 2016 /$ $\mathrm{mm}^{2}$ were $76.4 \%$ and $63.5 \%$, respectively. These differences in OS were not significant based on the Log rank test ( $p=0.096$; Figure 2E). Similarly, the OS probability analysis revealed that the five-year OS rates for patients in subgroups stratified by $\mathrm{CD} 8 / \mathrm{CD} 3>0.592$ and $\leq 0.592$ were $76.5 \%$ and $64.5 \%$, respectively. These differences in OS were also not significant ( $p=0.056$; Figure $2 \mathrm{G}$ ): however, the OS probability analysis revealed that the five-year OS rates for patients in subgroups stratified by CD $8+$ cell densities $>1125 / \mathrm{mm}^{2}$ and $\leq 1125 / \mathrm{mm}^{2}$ were $77.2 \%$ and $62.7 \%$, respectively. These differences in OS were statistically significant ( $p=0.021$; Figure 2F). Likewise, the OS probability analysis revealed that the five-year OS rates for patients in subgroups stratified by scores 2,1 and 0 were $79.1 \%, 71.3 \%$ and $61.2 \%$, respectively, and these differences were also significant $(p=0.034$; Figure 2H).

A Cox proportional hazards model was also used to evaluate the association of the studied parameters and various clinicopathological factors with OS. When using the $\mathrm{CD} 3$ counts, the $\mathrm{CD} 8$ counts, and the $\mathrm{CD} 8 / \mathrm{CD} 3$ ratio as the continuous variables, univariate analysis demonstrated that the hazard ratio [95\% confidence interval] was $1.00[0.999-1.000](p=0.040), 1.00$ [0.999-1.000] $(p=0.037)$, and $0.859[0.206-3.575](p=0.834)$, respectively. Univariate analysis revealed that more advanced overall stages, perineural invasion, extranodal extension, tumor depth ( $>5 \mathrm{~mm})$, lower CD8 counts, and lower scores (score 0 vs 2 ) indicated poorer OS (Table 3 ). To validate the independent prognostic factors, we performed a multivariate analysis that consisted of all the risk factors listed in the current AJCC staging as well as perineural invasion, which has long been regarded as a risk factor for locoregional recurrence and poorer survival. The results indicated that extranodal extension and lower scores (score 0 vs 2 ) were both independent factors associated with OS $(p<0.0001$ and $p=0.0369$, respectively; Table 3$)$.

\section{Discussion}

$\mathrm{T}$ cells mediate cellular immunity and are categorized into CD4+ helper T cells and CD8+ cytotoxic T cells, with the latter releasing cytotoxic granules to kill tumor cells. ${ }^{14}$ The role of TILs has been studied in melanoma for decades, but its prognostic importance was only recently emphasized in colorectal cancer. ${ }^{15,16}$ Galon et al quantified the densities of CD8+ and CD3+ TILs at both the invasive margin and tumor center in whole sections of colorectal cancer tumor tissue slides and found that the extent of $\mathrm{CD} 8+$ and $\mathrm{CD} 3+$ TILs at the tumor center and invasive margin was more predictive of clinical outcome than traditional TNM staging. ${ }^{17}$ In addition to colorectal cancer, extensive tumor lymphocyte infiltration is also associated with better overall survival and disease-free survival in non-small cell lung cancer. ${ }^{9}$ The total CD8+ cell count was also associated with improved cancer-specific survival in 1344 breast cancer patients: ${ }^{10}$ however, in head and neck cancer, a standardized method to evaluate tumor-infiltrating 


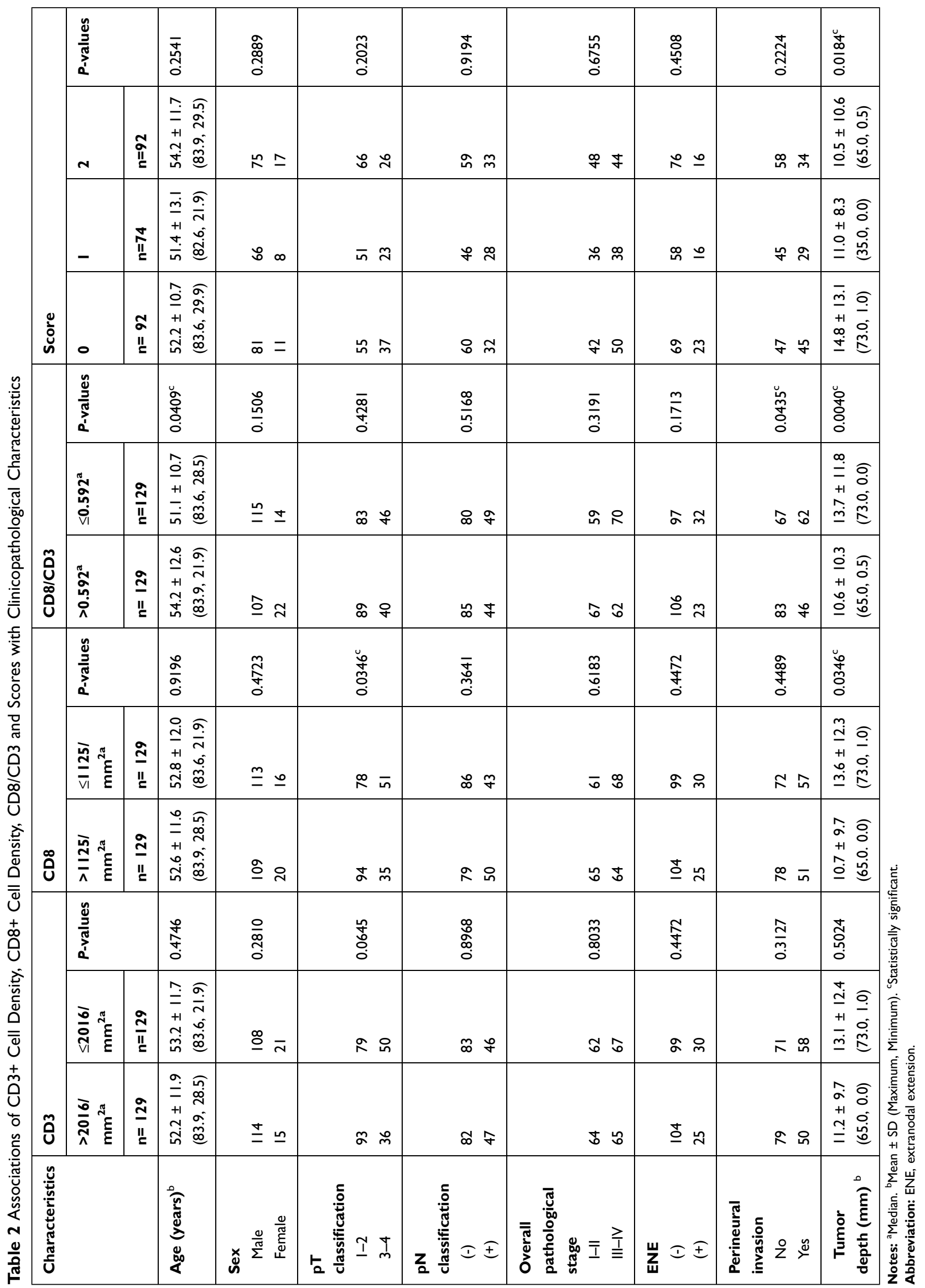



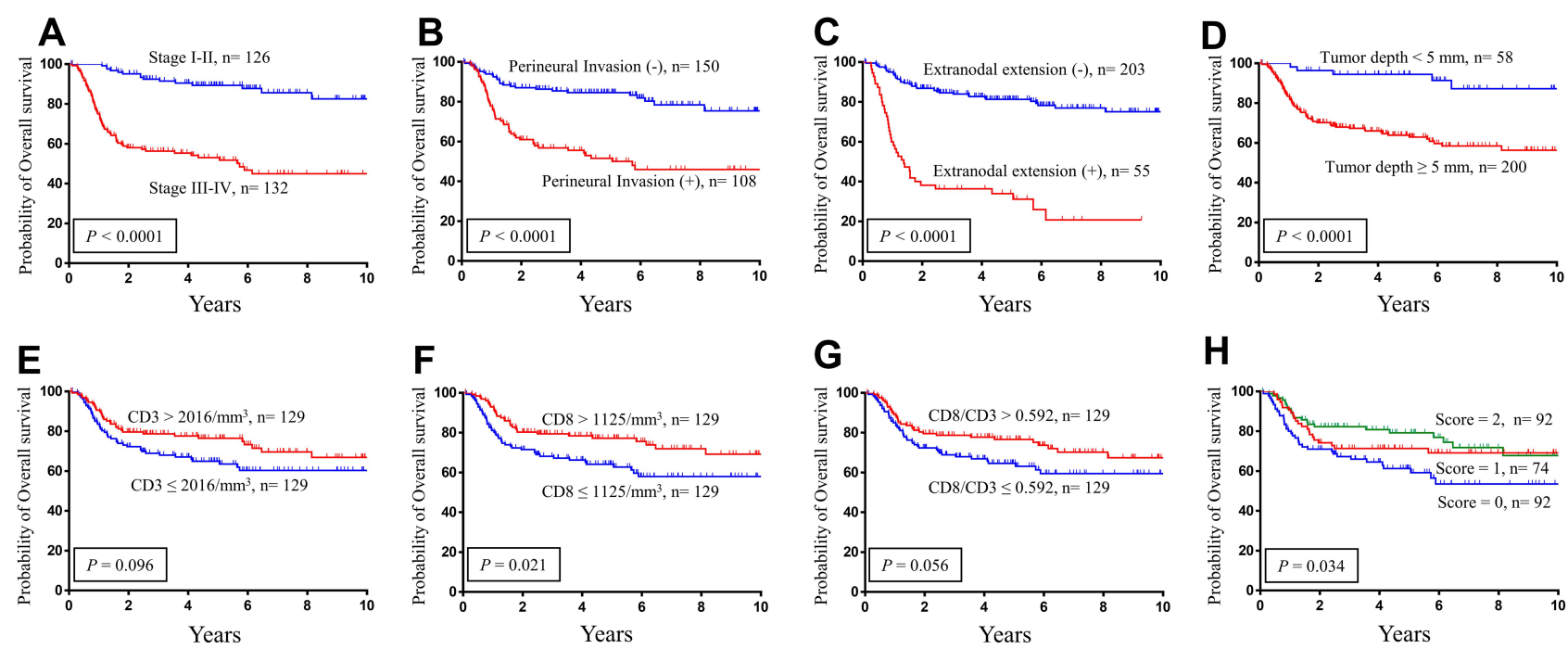

Figure 2 The Kaplan-Meier plots demonstrates the association of (A) overall stage, (B) Perineural invasion, (C) Extranodal extension, (D) Tumor depth, (E) CD3+ tumor-infiltrating leukocytes (TILs), (F) CD8+ TILs, (G) CD8/CD3, and (H) Scores with patient prognoses.

lymphocytes is still lacking. Here, digital image analysis was used for the first time to evaluate CD3+/CD8+ TILs at tumor invasive margins in a large homogeneous cohort of TSCC patients. The results showed that the CD8+ TIL density was significantly associated with more aggressive phenotypes and OS.

The spatial distribution of TILs can be divided into those in the tumor (epithelial component, intratumoral) or in the stroma (stromal component). Prior studies usually divide the TILs into these two components and many have focused on the intratumoral (epithelial) component. The stromal component has not been clearly defined in the literature and may or may not consist of the invasive margin, which may underlie the inconsistent conclusions on the TIL component that plays a more important role regarding patient survival. The role of TILs at the invasive margin has been rarely studied in the setting of head and neck cancers. The importance of the invasive margin has already been emphasized in other types of cancer, for example in colorectal cancer. ${ }^{11}$ In addition, the risk model proposed by Brandwein-Gensler et al consists of perineural invasion, lymphocytic host response, and a worse pattern of invasion. ${ }^{18}$ The latter two parameters actually represent the evaluation of the invasive margin. We hypothesize that the invasive margin is the site where TILs mostly interact with the tumor cells for TSCC and other types of cancer as well. In addition, previous studies did not conclude whether epithelial or stromal CD8+ TILs are a better predictor associated with the survival of OSCC patients. Therefore, we focused on the role of
TILs at the invasive margin in our large, homogeneous population.

Prior studies have demonstrated that TILs in different compartments are associated with patient prognosis and/or treatment outcomes in OSCC. Distel et al found that in the low-risk group, higher numbers of intraepithelial CD8+ TILs were associated with better survival. ${ }^{19}$ Oguejiofor et al found that the stromal infiltration of CD8+ TILs was associated with improved outcomes in HPV-related oropharyngeal SCC. ${ }^{20}$ More recently, another study demonstrated that a high density of CD8+ TILs in the tumor center (probably representing the intratumoral TILs) was associated with increased OS in OSCC. ${ }^{21}$ Lechner et al investigated TILs in both the tumor centers and invasive margins, but little information was provided in their results regarding the spatial distribution and

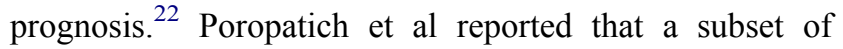
cases (HPV-positive) displayed a unique TIL pattern consisting of a circumferential peritumoral population of $\mathrm{T}$ cells, which was absent in the HPV-negative cases in 40 oral tongue and oropharyngeal cancer patients. ${ }^{23}$ In breast cancer, TILs also contribute to patient response to neoadjuvant chemotherapy. Khoury et al found that intratumoral TILs were an independent prognosticator of a pathological complete response in the luminal type of breast cancer, while both intratumoral and stromal TILs were independent predictors of a pathological complete response in triple negative breast cancer. ${ }^{24}$

The conflicting results may be due to the following: (1) the studied populations were not homogeneous, ranging 
Table 3 Univariate and Multivariate Analyses of Overall Survival in Patients with Oral Cavity Squamous Cell Carcinoma After Treatment

\begin{tabular}{|c|c|c|c|c|c|c|}
\hline \multirow[t]{2}{*}{ Variables } & \multicolumn{3}{|l|}{ Univariate } & \multicolumn{3}{|l|}{ Multivariate } \\
\hline & Hazard Ratio & $95 \% \mathrm{Cl}$ & $P$-value & Hazard Ratio & $95 \% \mathrm{Cl}$ & $P$-value \\
\hline Age (years) & 1.002 & $0.984-1.020$ & 0.8445 & 1.011 & $0.990-1.033$ & 0.2933 \\
\hline \multicolumn{7}{|l|}{ Sex } \\
\hline Male & Reference & & & Reference & & \\
\hline Female & 0.834 & $0.430-1.619$ & 0.5921 & 1.033 & $0.514-2.076$ & 0.9279 \\
\hline \multicolumn{7}{|c|}{ Overall pathological stage } \\
\hline I-II & Reference & & & Reference & & \\
\hline III-IV & 5.449 & $3.145-9.440$ & $<0.0001^{b}$ & 2.572 & $1.321-5.007$ & 0.0054 \\
\hline \multicolumn{7}{|c|}{ Perineural invasion } \\
\hline No & Reference & & & & & \\
\hline Yes & 1.926 & I. $.453-2.553$ & $<0.0001^{b}$ & & & \\
\hline \multicolumn{7}{|l|}{ ENE } \\
\hline$(-)$ & Reference & & & Reference & & \\
\hline$(+)$ & 5.952 & $3.799-9.325$ & $<0.0001^{b}$ & 3.084 & $1.827-5.206$ & $<0.0001^{b}$ \\
\hline \multicolumn{7}{|c|}{ Tumor depth (mm) } \\
\hline$\leq 5 \mathrm{~mm}^{\mathrm{a}}$ & Reference & & & Reference & & \\
\hline$>5 \mathrm{~mm}^{\mathrm{a}}$ & 5.460 & $2.206-13.513$ & $0.0002^{b}$ & 2.332 & $0.872-6.234$ & 0.0916 \\
\hline \multicolumn{7}{|l|}{ CD3 } \\
\hline$<2016 / \mathrm{mm}^{2 \mathrm{a}}$ & Reference & & & & & \\
\hline$>2016 / \mathrm{mm}^{2 \mathrm{a}}$ & 0.690 & $0.444-1.07 \mid$ & 0.0980 & & & \\
\hline \multicolumn{7}{|l|}{ CD8 } \\
\hline$<1125 / \mathrm{mm}^{2 \mathrm{a}}$ & Reference & & & & & \\
\hline$>1125 / \mathrm{mm}^{2 a}$ & 0.597 & $0.383-0.930$ & $0.0227^{b}$ & & & \\
\hline \multicolumn{7}{|l|}{ CD8/CD3 } \\
\hline$<0.592^{\mathrm{a}}$ & Reference & & & & & \\
\hline$>0.592^{\mathrm{a}}$ & 0.652 & $0.419-1.015$ & 0.0580 & & & \\
\hline \multicolumn{7}{|l|}{ Score } \\
\hline 2 & Reference & & & Reference & & \\
\hline 1 & 0.657 & 0.388-I.III & 0.1174 & 0.718 & $0.420-1.229$ & 0.2270 \\
\hline 0 & 0.512 & $0.30 I-0.87 I$ & $0.0135^{\mathrm{b}}$ & 0.559 & $0.324-0.965$ & $0.0369^{b}$ \\
\hline
\end{tabular}

Notes: a Median. ${ }^{\text {b}}$ Statistically significant

Abbreviations: $\mathrm{Cl}$, confidence interval; $\mathrm{ENE}$, extranodal extension.

from HPV-related oropharyngeal cancers to oral cancers that probably includes oral tongue, base of the tongue, gingiva, and buccal mucosa; (2) the definition of the stromal and epithelial compartments was not clear. The epithelial compartment might be defined as TILs infiltrating in the tumor, but the TILs in the stromal compartment include all the TILs distributed from micrometers to millimeters away from the tumor; and (3) the number of patients included in each study differed, ranging from tens to hundreds of patients. The definition of the epithelial and stromal compartment of TILs differed among previous studies, and the TILs in the invasive margin of OSCC were only studied by Lechner et al and Zhou et al with relatively small numbers of patients. ${ }^{21,22}$ Therefore, the current study aimed to investigate the association of TILs at the invasive margins in a large, homogeneous population of TSCC.

Based on our preliminary observation, in OSCC, tumor-infiltrating immune cells were more likely to be concentrated at the invasive margins than at the tumor centers. Thus, in the current study, we did not follow the methods used by previous studies to measure the TILs in 
the tumor cores. ${ }^{17}$ The current study demonstrated the superiority of evaluating TILs at the invasive margins to those at the tumor centers. The results showed that lower $\mathrm{CD} 8+$ cell counts and $\mathrm{CD} 8$ to $\mathrm{CD} 3$ ratio at the invasive margins were associated with increased tumor depths in TSCC. These findings might be explained by the cytotoxic activity of CD8+ TILs at the invasive margin, which could impede the growth of tumor cells and hence enhance antitumor immunity in the microenvironment. Similarly, in a previous study of malignant melanoma, TIL measurements revealed that the presence of sparse TILs and tumor thickness were significant and independent predictive histologic factors for prognosis. ${ }^{25}$

Several publications have discussed the prognostic role of TILs in OSCC. Fang et al found that a higher CD8 and CD57 expression in TILs in the tumor stroma was correlated with longer OS in 78 patients with oral cavity cancer. ${ }^{6}$ Cho et al evaluated the intratumoral and peritumoral densities of CD4 +/CD8+ TILs, PD-L1 expression in tumor cells, and tumorassociated fibroblasts in 45 OSCC patients and found that peritumoral TILs were significantly associated with tumor size, lymph node metastasis and clinical stage. ${ }^{26}$ Shimizu et al evaluated the density of CD8+ TILs in five compartments of 193 OSCC patients and found that a high stromal $\mathrm{CD}^{+} \mathrm{TIL}$ density at the tumor periphery and high parenchymal $\mathrm{CD} 8^{+}$TIL density at the invading edge were independent prognostic markers for recurrence-free survival and OS. $^{7}$ Wolf et al evaluated different subsets of tumorinfiltrating immune cells, including CD8+ TILs, in 39 OSCC patients and found that CD68+ tumor-associated macrophages rather than CD8+ TILs were associated with poorer overall survival, demonstrating conflicting results with other studies. ${ }^{5-8}$ Additionally, most previous studies evaluated TIL densities by subjective measurement under microscopy with various fields of interest within OSCC specimens. In our investigation, we identified two main obstacles that can reduce the reliability and reproducibility of the visual assessment of TIL counts under microscopy. First, at the invasive margin, TILs tend to form aggregates, making it difficult to accurately determine the actual numbers of CD8+ TILs. Second, the staining intensities of CD3 and CD8 are usually homogeneous and strong, and thus a weaker and heterogeneous staining pattern could be neglected. To overcome these potential biases in the current study, we adopted digital image analysis at the invasive margins of TSCC tumors and found that this method was more objective and efficient in assessing the density of TILs at invasive margins.
OSCCs consist of tumors originating from the buccal mucosa, gingiva, hard palate, retromolar, floor of mouth, and tongue. Previous studies enrolled patients with oral cavity squamous cell carcinoma from various sublocations. ${ }^{6-8}$ In addition to tumors in the tongue, tumors originating from other sublocations might encroach upon physical barriers, such as skin or bone. These physical barriers might impede the growth of tumor cells and therefore lead to an underestimation of the density of TILs at the invasive front. To achieve a more subjective and scientific evaluation, the current study only enrolled TSCC patients to investigate the effect of CD8+ TILs at the invasive margin on clinicopathological features and survival.

\section{Conclusions}

This study demonstrated the association of CD8+ and CD3 + TILs with overall survival and tumor depth in a large homogeneous cohort of TSCC patients using a digital image analysis method. Along with extranodal extension, a low abundance of CD8+ and CD3+ TILs predicted a shorter OS in TSCC. Together, the results emphasize the importance of CD8+ TILs in the prognosis of TSCC, and the application of simple immunohistochemistry combined with digital image analysis to provide a novel parameter to supplement current TNM staging for TSCC patient stratification.

\section{Data Sharing Statement}

Datasets used for analysis for this study are available for review, without compromising patient confidentiality, upon reasonable request.

\section{Ethics Approval and Consent to Participate}

The study protocol was approved by the Institutional Review Board of Chang Gung Memorial Hospital, Taiwan.

\section{Consent for Publication}

Written informed consent was obtained from each patient for study participation and publication.

\section{Acknowledgments}

The authors thank all the members of the Cancer Center, Chang Gung Memorial Hospital, for their invaluable help and Prof. David Chwei-Chin Chuang for generously providing Image-Pro Premier 9.1 software. 


\section{Author Contributions}

KPC: Conception of study, study design, execution, acquisition of data, analysis and interpretation. YH: Conception of study, execution and acquisition of data. CL: Acquisition of data and analysis. HKK: Acquisition of data and interpretation. SYH: Acquisition of data and analysis. HJK: Acquisition of data, execution and analysis. YCH: Acquisition of data and analysis. YLC: Acquisition of data and interpretation. All authors: Have drafted or written, or substantially revised or critically reviewed the article; have agreed on the journal to which the article will be submitted; reviewed and agreed on all versions of the article before submission, during revision, the final version accepted for publication, and any significant changes introduced at the proofing stage; agree to take responsibility and be accountable for the contents of the article.

\section{Funding}

This study was supported by grants (CMRPG3A1233, CMRPG3H0851 and CMRPG3J1252 from Chang Gung Memorial Hospital) and MOST 108-2314-B-182A-108MY3 from Ministry of Science and Technology, Taiwan.

\section{Disclosure}

The authors report no conflicts of interest for this work.

\section{References}

1. Hsu WL, Yu KJ, Chiang CJ, Chen TC, and Wang CP. Head and neck cancer incidence trends in Taiwan, $1980 \sim 2014$. Int J Head Neck Sci. 2017;1(3):180-189. doi:10.6696/IJHNS.2017.0103.05

2. De Paz D, Kao HK, Huang Y, Chang KP. Prognostic stratification of patients with advanced oral cavity squamous cell carcinoma. Curr Oncol Rep. 2017;19(10):65. doi:10.1007/s11912-017-0624-3

3. De Paz D, Chang KP, Kao HK, et al. Clinical implications of tumor-associated tissue eosinophilia in tongue squamous cell carcinoma. Laryngoscope. 2019;129(5):1123-1129. doi:10.1002/lary.27413

4. Fan KH, Lin CY, Kang CJ, et al. Combined-modality treatment for advanced oral tongue squamous cell carcinoma. Int $J$ Radiat Oncol Biol Phys. 2007;67(2):453-461. doi:10.1016/j.ijrobp.2006.06.026

5. Chen W-Y, Wu C-T, Wang C-W, et al. Prognostic significance of tumor-infiltrating lymphocytes in patients with operable tongue cancer. Radiat Oncol. 2018;13(1):157. doi:10.1186/s13014-018-1099-6

6. Fang J, Li X, Ma D, et al. Prognostic significance of tumor infiltrating immune cells in oral squamous cell carcinoma. BMC Cancer. 2017;17 (1):375. doi:10.1186/s12885-017-3317-2

7. Shimizu S, Hiratsuka H, Koike K, et al. Tumor-infiltrating CD8(+) T-cell density is an independent prognostic marker for oral squamous cell carcinoma. Cancer Med. 2019;8(1):80-93. doi:10.1002/cam4.1889

8. Wolf GT, Chepeha DB, Bellile E, Nguyen A, Thomas D, McHugh J. Tumor infiltrating lymphocytes (TIL) and prognosis in oral cavity squamous carcinoma: a preliminary study. Oral Oncol. 2015;51 (1):90-95. doi:10.1016/j.oraloncology.2014.09.006

9. Brambilla E, Le Teuff G, Marguet S, et al. Prognostic effect of tumor lymphocytic infiltration in resectable non-small-cell lung cancer. J Clin Oncol. 2016;34(11):1223-1230. doi:10.1200/JCO.2015.63.0970
10. Mahmoud SMA, Paish EC, Powe DG, et al. Tumor-infiltrating CD8+ lymphocytes predict clinical outcome in breast cancer. J Clin Oncol. 2011;29(15):1949-1955. doi:10.1200/jco.2010.30.5037

11. Pages F, Mlecnik B, Marliot F, et al. International validation of the consensus immunoscore for the classification of colon cancer: a prognostic and accuracy study. Lancet. 2018;391(10135):2128-2139. doi:10.1016/S0140-6736(18)30789-X

12. Lee SL, Cabanero M, Hyrcza M, et al. Computer-assisted image analysis of the tumor microenvironment on an oral tongue squamous cell carcinoma tissue microarray. Clin Transl Radiat Oncol. 2019;17: (32-39. doi:10.1016/j.ctro.2019.05.001

13. Mlecnik B, Bindea G, Kirilovsky A, et al. The tumor microenvironment and immunoscore are critical determinants of dissemination to distant metastasis. Sci Transl Med. 2016;8(327):327ra326. doi:10.1126/scitranslmed.aad6352

14. Binnewies M, Roberts EW, Kersten K, et al. Understanding the tumor immune microenvironment (TIME) for effective therapy. Nat Med. 2018;24(5):541-550. doi:10.1038/s41591-018-0014-x

15. Hansen MG, McCarten AB. Tumor thickness and lymphocytic infiltration in malignant melanoma of the head and neck. Am J Surg. 1974;128(4):557-561. doi:10.1016/0002-9610(74)90275-x

16. Taube JM, Galon J, Sholl LM, et al. Implications of the tumor immune microenvironment for staging and therapeutics. Mod Pathol. 2018;31(2):214-234. doi:10.1038/modpathol.2017.156

17. Galon J, Mlecnik B, Bindea G, et al. Towards the introduction of the 'immunoscore' in the classification of malignant tumours. $J$ Pathol. 2014;232(2):199-209. doi:10.1002/path.4287

18. Brandwein-Gensler M, Smith RV, Wang B, et al. Validation of the histologic risk model in a new cohort of patients with head and neck squamous cell carcinoma. Am J Surg Pathol. 2010;34(5):676-688. doi:10.1097/PAS.0b013e3181d95c37

19. Distel LV, Fickenscher R, Dietel K, et al. Tumour infiltrating lymphocytes in squamous cell carcinoma of the oro- and hypopharynx: prognostic impact may depend on type of treatment and stage of disease. Oral Oncol. 2009;45(10):e167-e174. doi:10.1016/j.oraloncology.2009.05.640

20. Oguejiofor K, Hall J, Slater C, et al. Stromal infiltration of CD8 T cells is associated with improved clinical outcome in HPV-positive oropharyngeal squamous carcinoma. $\mathrm{Br} \quad \mathrm{J}$ Cancer. 2015;113 (6):886-893. doi:10.1038/bjc.2015.277

21. Zhou C, Wu Y, Jiang L, et al. Density and location of CD3(+) and $\mathrm{CD} 8(+)$ tumor-infiltrating lymphocytes correlate with prognosis of oral squamous cell carcinoma. J Oral Pathol Med. 2018;47 (4):359-367. doi:10.1111/jop.12698

22. Lechner A, Schlosser H, Rothschild SI, et al. Characterization of tumor-associated T-lymphocyte subsets and immune checkpoint molecules in head and neck squamous cell carcinoma. Oncotarget. 2017;8(27):44418-44433. doi:10.18632/oncotarget.17901

23. Poropatich K, Hernandez D, Fontanarosa J, et al. Peritumoral cuffing by T-cell tumor-infiltrating lymphocytes distinguishes HPV-related oropharyngeal squamous cell carcinoma from oral cavity squamous cell carcinoma. J Oral Pathol Med. 2017;46(10):972-978. doi:10.1111/ jop. 12605

24. Khoury T, Nagrale V, Opyrchal M, Peng X, Wang D, Yao S. Prognostic significance of stromal versus intratumoral infiltrating lymphocytes in different subtypes of breast cancer treated with cytotoxic neoadjuvant chemotherapy. Appl Immunohistochem Mol Morphol. 2018;26(8):523-532. doi:10.1097/PAI.0000000000000466

25. Clemente CG, Mihm MC, Bufalino R, Zurrida S, Collini P, Cascinelli N. Prognostic value of tumor infiltrating lymphocytes in the vertical growth phase of primary cutaneous melanoma. Cancer. 1996;77(7):1303-1310. doi:10.1002/(sici)1097-0142(19960401) 77:7<1303::Aid-cncr12>3.0.Co;2-5

26. Cho Y-A, Yoon H-J, Lee J-I, Hong S-P, Hong S-D. Relationship between the expressions of PD-L1 and tumor-infiltrating lymphocytes in oral squamous cell carcinoma. Oral Oncol. 2011;47 (12):1148-1153. doi:10.1016/j.oraloncology.2011.08.007 


\section{Publish your work in this journal}

Cancer Management and Research is an international, peer-reviewed open access journal focusing on cancer research and the optimal use of preventative and integrated treatment interventions to achieve improved outcomes, enhanced survival and quality of life for the cancer patient.
The manuscript management system is completely online and includes a very quick and fair peer-review system, which is all easy to use. Visit http://www.dovepress.com/testimonials.php to read real quotes from published authors. 